\title{
Medication compliance in Singaporean patients with Alzheimer's disease
}

\author{
Zheng Kang $\underline{\mathrm{Lum}}^{1}$, BSc(Pharm)(Hons), Ma Serrie P $\underline{\text { Suministrado }}^{1,2}$, MD, $N \underline{\text { Venketasubramanian }}{ }^{2,3}$, MBBS, MMed, \\ M Kamran $\underline{\mathrm{kram}}^{4}$, MD, PhD, Christopher $\underline{\text { Chen }}^{1,2}$, BMBCh, FRCP
}

INTRODUCTION Singapore has a rapidly ageing population and an increasing prevalence of Alzheimer's disease (AD). Compliance to AD medications is associated with treatment effectiveness. We investigated compliance to acetylcholinesterase inhibitors (AChEIs) and N-methyl-D-aspartate (NMDA) receptor antagonist and treatment persistence among patients seen at the General Memory Clinic of National University Hospital, Singapore. We also identified the reasons for non-compliance.

METHODS Patients seen at the General Memory Clinic between 1 January 2013 and 31 December 2014, who were prescribed AChEls and NMDA receptor antagonist, were included in this retrospective cohort study. Non-compliance to medications was indirectly measured by failure to renew prescription within 60 days of the last day of medication supplied by the previous prescription. The reasons for non-compliance were identified.

RESULTS A total of 144 patients were included. At one year, 107 patients were compliant to AD medications, while 37 patients were non-compliant. Around $60 \%$ of the non-compliant patients discontinued the use of AD medications within the first six months, and the mean persistent treatment period among this group of patients was $10.3 \pm 3.5$ months. The main reason for non-compliance was patients' and caregivers' perception that memory loss was of lower priority than other coexisting illnesses. Other reasons for non-compliance included side effects of medications (18.9\%), perceived ineffectiveness of treatment (16.2\%), inability to attend clinic (5.4\%) and high cost of medications $(2.7 \%)$.

CONCLUSION Our findings suggest that the reasons for medication non-compliance can be identified early. Better compliance may be achieved through a multidisciplinary approach to patient education.

Keywords: acetylcholinesterase inhibitors, Alzheimer's disease, compliance, NMDA receptor antagonist

\section{INTRODUCTION}

In 2015, it was estimated that around 50 million individuals suffered from dementia globally, ${ }^{(1)}$ with Alzheimer's disease (AD) being the most common cause of dementia. ${ }^{(2)}$ This number is projected to increase to 135 million by $2050 .^{(2)}$ Singapore has one of the fastest ageing populations in the Asia Pacific region and the prevalence of dementia is expected to increase substantially. ${ }^{(3)}$ Dementia is a major healthcare challenge, as it is a leading cause of disability and high healthcare cost. ${ }^{(3)}$ The main class of drug approved for the management of mild to severe dementia due to $\mathrm{AD}$ is the acetylcholinesterase inhibitors (AChEls), ${ }^{(4)}$ which antagonise the action of acetylcholinesterase ${ }^{(5)}$ and target the cholinergic deficit in $\mathrm{AD},{ }^{(6)}$ hence improving mood, cognition and behaviour. Memantine, an N-methyl-D-aspartate (NMDA) receptor antagonist, is recommended for use in $\mathrm{AD}$ patients with moderate to severe dementia.

Compliance to the use of $\mathrm{AD}$ medications is crucial to achieving maximal treatment efficacy. ${ }^{(7)}$ However, medication compliance in $\mathrm{AD}$ patients is a problem due to their diminished cognition. ${ }^{(8)}$ Furthermore, the side effects and high cost of AD medications makes compliance even more challenging. In an Austrian study, more than $50 \%$ of patients with dementia discontinued the use of AChEls within 12 months of therapy initiation. ${ }^{(9)}$ Similarly, a Canadian study reported a noncompliance rate of $46 \%$ for galantamine, $54 \%$ for donepezil and $60 \%$ for rivastigmine after one year of initiation of AChEls. ${ }^{(4)}$ Borah et al found that more than $40 \%$ of AD patients were non-compliant to medications, and attributed it to overall pill burden (odds ratio [OR] 1.192, $\mathrm{p}<0.001$ ). ${ }^{(10)}$ A recent review identified several determinants of non-compliance to $A D$ medications, including patients' belief that AD is age-related, medication side effects and caregivers' unrealistic expectations of treatment benefits. ${ }^{(1)}$ Medication non-compliance has critical negative implications on achieving optimal treatment outcome and indirectly imposes significant economic cost to the healthcare system. ${ }^{(12)}$ In addition, medication non-compliance among AD patients was found to be associated with a higher risk of hospital admission. ${ }^{(13)}$

As no local data on medication compliance in $A D$ is available, this study aimed to identify the reasons for noncompliance to medications and estimate the time-point of treatment discontinuation among AD patients in Singapore. We opine that treatment continuation and medication compliance are influenced by a country's unique cultural and social factors. Hence, this study may provide a foundation to improve the overall management of $A D$ in Singapore.

${ }^{1}$ Department of Pharmacology, National University of Singapore, ${ }^{2}$ Memory Ageing and Cognition Centre, National University Health System, ${ }^{3}$ Raffles Neuroscience Centre, Raffles Hospital, ${ }^{4}$ Departments of Neurology and Epidemiology, Erasmus Medical Center Rotterdan, the Netherlands 


\section{METHODS}

Participants of this study were drawn from patients whose first visit to the General Memory Clinic, National University Hospital, Singapore, was between 1 January 2013 and 31 December 2014. All patients were diagnosed using clinical and neuropsychological assessment results at a weekly consensus meeting attended by clinicians and neuropsychologists. This study included patients who had been diagnosed with AD and prescribed with AChEls and/or NMDA receptor antagonist. Information on the study population, such as demographics, education level, clinical history and medications, was extracted from hospital records. Compliance to $\mathrm{AD}$ medications was tracked for at least one year. Telephone calls were made to the caregiver of patients who defaulted on appointment without specific reasons to understand their reasons for non-compliance to medication. The duration of treatment persistence was also recorded for this group of patients to determine the proportion of patients who persisted with treatment for $<6$ months, 6-12 months and $>12$ months after their first visit to the General Memory Clinic.

The definition of medication compliance in this study aligns to that of the World Health Organization (WHO), which is the degree of conformity to the recommendations on medications prescribed with respect to the timing, frequency and dosage by a healthcare provider. ${ }^{(14)}$ Non-compliance to medication in this study was indirectly measured by the failure to renew prescription within 60 days of the last day of medication supplied by the previous prescription. The duration of 60 days was selected, as it is the average follow-up duration between visits for each patient. Several studies have also used failure to renew a prescription during a 60-day follow-up period as an indicator of non-compliance. ${ }^{(15-17)}$ Patients discharged from regular follow-up with the General Memory Clinic to another clinical provider or discharged to outpatient community care were considered to be compliant to medication use. Treatment persistence in the study was defined as the duration for which a patient complied with the treatment without defaulting at least one scheduled follow-up appointment with the General Memory Clinic.

Factors for non-compliance to AD medications were reported in accordance with the five dimensions classified by the WHO: ${ }^{(14)}$ (1) social and economic factors such as illiteracy, unstable living conditions and high cost of medications; (2) healthcare team- and system-related factors such as healthcare provider-patient relationship and healthcare costs; (3) condition-related factors such as severity of symptoms and comorbidities; (4) therapyrelated factors such as complexity of medical regimen, duration of treatment and previous treatment failures; and (5) patient-related factors such as age, gender, resources, knowledge, perception, attitudes and beliefs.

Data analysis was performed using the IBM SPSS Statistics version 22.0 (IBM Corp, Armonk, NY, USA). All statistical tests were two-tailed with a significance level $(\alpha)$ of 0.05 . Univariate analysis was performed (chi-square test for categorical and independent samples; $t$-test for continuous variables) to explore associations of demographic characteristics and compliance to medication use among AD patients. The following variables were analysed: age; gender; ethnicity; Mini Mental State Examination (MMSE) scores; and Montreal Cognitive Assessment (MoCA) scores. Approval to conduct this study was obtained from the National Healthcare Group Ethics Review Board (DomainSpecific Review Board).

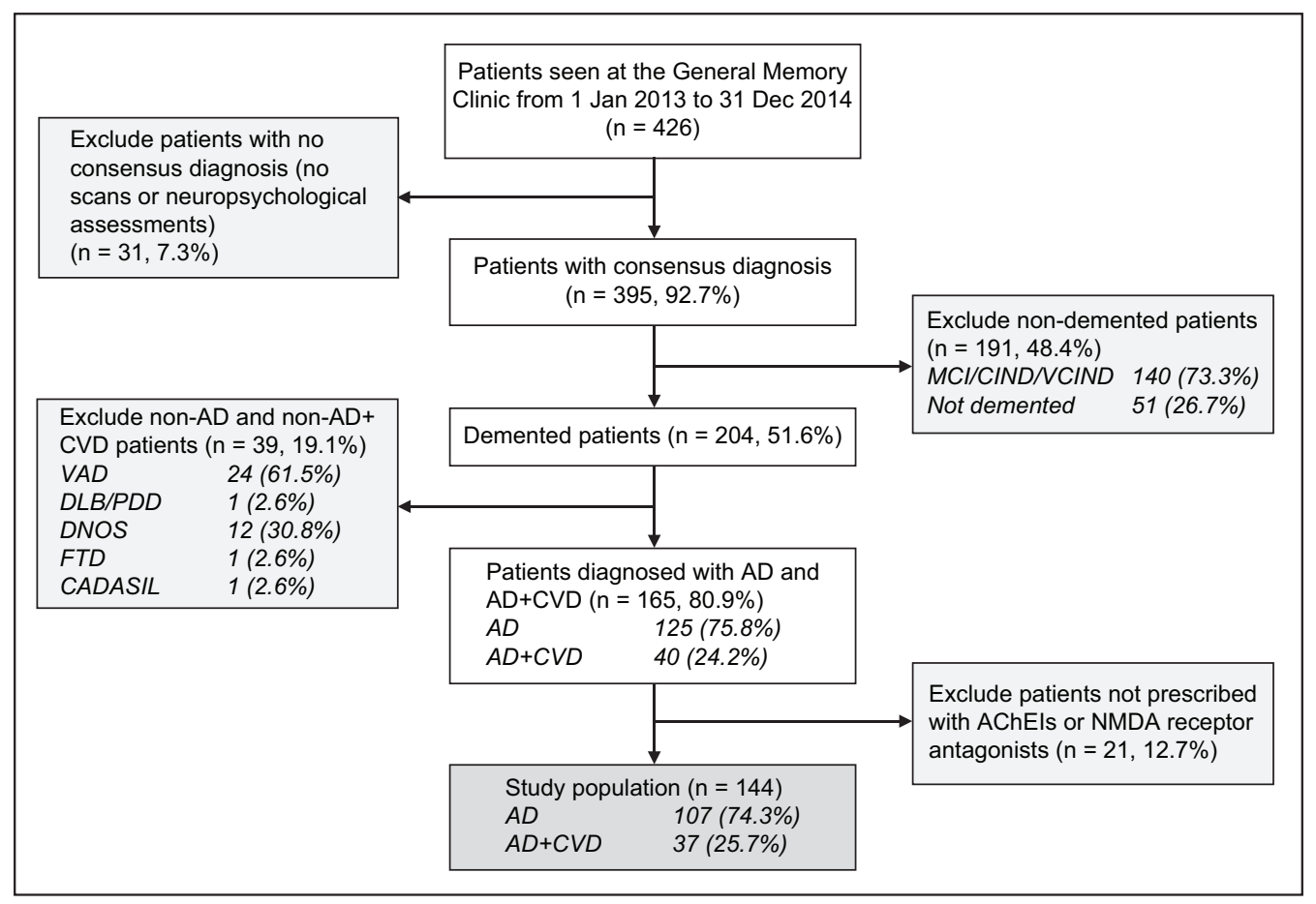

Fig. 1 Flow chart for the inclusion of patients in the study. AChEls: acetylcholinesterase inhibitors; AD: Alzheimer's disease; CADASIL: cerebral autosomal dominant arteriopathy with subcortical infarcts and leukoencephalopathy; CIND: cognitive impaired, no dementia; DLB: dementia with Lewy bodies; DNOS: dementia with no specified cause; FTD: fronto-temporal dementia; MCI: mild cognitive impairment; NMDA: N-methyl-D-aspartate; PDD: Parkinson's disease with dementia; VAD: vascular dementia; VCIND: vascular cognitive impairment, no dementia 
Table I. Characteristics of patients included in the study $(n=144)$.

\begin{tabular}{|c|c|c|c|c|}
\hline \multirow[t]{2}{*}{ Characteristic } & \multicolumn{3}{|c|}{ Mean \pm SD/no. (\%) } & \multirow[t]{2}{*}{ p-value } \\
\hline & Total $(n=144)$ & Compliant $(n=107)$ & Non-compliant $(n=37)$ & \\
\hline Age (yr) & $76.9 \pm 7.9$ & $76.6 \pm 8.2$ & $77.1 \pm 7.8$ & 0.774 \\
\hline Gender & & & & 0.332 \\
\hline Male & $49(34.0)$ & $34(31.8)$ & $15(40.5)$ & \\
\hline Female & $95(66.0)$ & $73(68.2)$ & $22(59.5)$ & \\
\hline Ethnicity & & & & 0.200 \\
\hline Chinese & $114(79.2)$ & $89(83.2)$ & $25(67.6)$ & \\
\hline Malay & $17(11.8)$ & $10(9.3)$ & $7(18.9)$ & \\
\hline Indians & $9(6.3)$ & $5(4.7)$ & $4(10.8)$ & \\
\hline Others & $4(2.8)$ & $3(2.8)$ & $1(2.7)$ & \\
\hline Highest education* & & & & 0.818 \\
\hline Primary and below & 99 (78.6) & $73(78.5)$ & $26(78.8)$ & \\
\hline Secondary & $19(15.1)$ & $15(16.1)$ & $4(12.1)$ & \\
\hline Tertiary and above & $8(6.3)$ & $5(5.4)$ & $3(9.1)$ & \\
\hline MMSE & $14.6 \pm 4.9$ & $15.1 \pm 5.1$ & $13.2 \pm 4.0$ & 0.075 \\
\hline MoCA & $9.8 \pm 4.7$ & $10.3 \pm 4.7$ & $8.3 \pm 4.4$ & 0.065 \\
\hline Types of AD medications & & & & 0.774 \\
\hline Donepezil & $129(89.6)$ & $97(90.7)$ & $32(86.5)$ & \\
\hline Rivastigmine & $9(6.3)$ & $6(5.6)$ & $3(8.1)$ & \\
\hline Memantine & $6(4.2)$ & $4(3.7)$ & $2(5.4)$ & \\
\hline Formulations of medications & & & & 0.390 \\
\hline Donepezil tablet & $84(58.3)$ & $65(60.7)$ & $19(51.4)$ & \\
\hline Donepezil orodispersible & $19(13.2)$ & $11(10.3)$ & $8(21.6)$ & \\
\hline Rivastigmine patch & $26(18.1)$ & $21(19.6)$ & $5(13.5)$ & \\
\hline Rivastigmine capsule & $6(4.2)$ & $4(3.7)$ & $2(5.4)$ & \\
\hline Memantine tablet & $9(6.3)$ & $6(5.6)$ & $3(8.1)$ & \\
\hline Classification of AD & & & & 0.125 \\
\hline Mild & $12(8.3)$ & $11(10.3)$ & $1(2.7)$ & \\
\hline Moderate & $86(59.7)$ & $66(61.7)$ & $20(54.1)$ & \\
\hline Severe & 46 (31.9) & $30(28.0)$ & $16(43.2)$ & \\
\hline
\end{tabular}

*Due to missing data for highest education, total $n=126$, compliant $n=93$ and non-compliant $n=33$. AD: Alzheimer's disease; MMSE: Mini Mental Examination; MoCA: Montreal Cognitive Assessment; SD: standard deviation

\section{RESULTS}

A total of 426 patients had their first visit to the General Memory Clinic between 1 January 2013 and 31 December 2014. These patients were screened for eligibility and a total of 144 patients were included in the study (Fig. 1). The characteristics of the 144 patients are summarised in Table I. Altogether, 36 patients were discharged -28 patients to outpatient community care and eight from regular follow-up with the General Memory Clinic. The remaining 71 patients continued to be on regular follow-up with the General Memory Clinic. Of the 144 included patients, 107 (74.3\%) were compliant to the use of AD medications. Table II summarises the characteristics of the non-compliant patients ( $\mathrm{n}=37,25.7 \%$ ).

Factors influencing medication non-compliance included patient-related, therapy-related, condition-related and socioeconomic factors (Table III). One of the main patient-related factors identified was the perception that treatment was ineffective $(n=6)$. While several studies have reported significant positive associations between AD medication compliance and older age/male gender, ${ }^{(18)}$ our study did not find any such significant associations. Two patients were unable to attend the clinic for follow-up appointment, as they were bedridden.

For therapy-related factors, 7 (18.9\%) patients were noncompliant due to side effects from the AD medications: six patients reported side effects from donepezil and one patient from rivastigmine patch. Side effects of donepezil were generally gastrointestinal-related, including nausea, diarrhoea and bloatedness, while the side effect of rivastigmine patch was headache.

In terms of condition-related factors, 12 (32.4\%) of the non-compliant patients and their caregivers viewed a decline in memory as a natural phenomenon of ageing, and hence perceived memory loss as being less important than other comorbidities. Among these patients, many were diagnosed with concomitant cardiovascular diseases, such as hypertension, heart failure and atrial fibrillation, which were managed with several medications. 
Table II. Characteristics of non-compliant patients included in this study $(n=37)$.

\begin{tabular}{|c|c|c|c|c|}
\hline \multirow[t]{2}{*}{ Characteristic } & \multicolumn{3}{|c|}{ Mean \pm SD/no. (\%) } & \multirow[t]{2}{*}{ p-value } \\
\hline & Total $(n=37)$ & $A D(n=28)$ & $A D+C V D(n=9)$ & \\
\hline Age (yr) & $76.6 \pm 8.1$ & $76.3 \pm 8.2$ & $77.7 \pm 8.1$ & 0.664 \\
\hline Gender & & & & 0.784 \\
\hline Male & $15(40.5)$ & $11(39.3)$ & $4(44.4)$ & \\
\hline Female & $22(59.5)$ & $17(60.7)$ & $5(55.6)$ & \\
\hline Ethnicity & & & & 0.242 \\
\hline Chinese & $25(67.6)$ & $18(64.3)$ & $7(77.8)$ & \\
\hline Malay & $7(18.9)$ & $7(25.0)$ & $0(0.0)$ & \\
\hline Indians & $4(10.8)$ & $2(7.1)$ & $2(22.2)$ & \\
\hline Others & $1(2.7)$ & $1(3.6)$ & $0(0.0)$ & \\
\hline Highest education* & & & & 0.133 \\
\hline Primary and below & $26(78.8)$ & $19(79.2)$ & $7(77.8)$ & \\
\hline Secondary & $4(12.1)$ & $4(16.7)$ & $0(0.0)$ & \\
\hline Tertiary and above & $3(9.1)$ & $1(4.2)$ & $2(22.2)$ & \\
\hline MMSE & $13.2 \pm 4.0$ & $13.8 \pm 4.0$ & $11.6 \pm 3.9$ & 0.207 \\
\hline MoCA & $8.3 \pm 4.4$ & $8.4 \pm 4.2$ & $8.0 \pm 5.2$ & 0.862 \\
\hline Types of AD medications & & & & 0.411 \\
\hline Donepezil & $32(86.5)$ & $24(85.7)$ & $8(88.9)$ & \\
\hline Rivastigmine & $3(8.1)$ & $3(10.7)$ & $0(0.0)$ & \\
\hline Memantine & $2(5.4)$ & $1(3.6)$ & $1(11.1)$ & \\
\hline Formulations of medications & & & & 0.137 \\
\hline Donepezil tablet & $19(51.4)$ & $11(39.3)$ & $8(88.9)$ & \\
\hline Donepezil orodispersible & $8(21.6)$ & $8(28.6)$ & $0(0.0)$ & \\
\hline Rivastigmine patch & $5(13.5)$ & $5(17.9)$ & $0(0.0)$ & \\
\hline Rivastigmine capsule & $2(5.4)$ & $2(7.1)$ & $0(0.0)$ & \\
\hline Memantine tablet & $3(8.1)$ & $2(7.1)$ & $1(11.1)$ & \\
\hline Classification of AD & & & & 0.103 \\
\hline Mild & $1(2.7)$ & $0(0.0)$ & $1(11.1)$ & \\
\hline Moderate & $20(54.1)$ & $15(53.6)$ & $5(55.6)$ & \\
\hline Severe & $16(43.2)$ & $13(46.4)$ & $3(33.3)$ & \\
\hline
\end{tabular}

*Due to missing data for highest education, total $n=33$ and $A D n=24$. AD: Alzheimer's disease; CVD: cardiovascular disease; MMSE: Mini Mental Status Examination; MoCA: Montreal Cognitive Assessment; SD: standard deviation

Table III. Reasons for non-compliance to medication use $(n=37)$.

\begin{tabular}{|ll|}
\hline Reason & No. (\%) \\
\hline Socioeconomic factors & $1(2.7)$ \\
\hline \multicolumn{1}{|l|}{ High cost of medications } & \\
\hline Condition-related factors & $12(32.4)$ \\
\hline $\begin{array}{l}\text { Memory loss perceived as lower in priority compared } \\
\text { to other comorbidities }\end{array}$ & \\
\hline Therapy-related factors & $7(18.9)$ \\
\hline Side effects of medications & \\
\hline Patient-related factors & $6(16.2)$ \\
\hline Perception that treatment was not effective & $2(5.4)$ \\
\hline Inability to attend clinic & $9(24.3)$ \\
\hline Deceased &
\end{tabular}

As these cardiovascular comorbidities were considered more critical than the memory loss experienced in $A D$, these patients and their caregivers inevitably neglected AD treatment and compliance to AD medications. Among this group of patients, two were undergoing chemotherapy, which compounded their social and emotional burden. The main socioeconomic factor that contributed to non-compliance was high medication costs $(\mathrm{n}=$ $1,2.7 \%$ ). While other studies have found illiteracy and low level of education to be significantly associated with poor medication compliance, ${ }^{(19)}$ our study did not find such associations.

The mean duration of persistent treatment was $10.3 \pm$ 3.5 months. Out of the 144 patients, 114 (79.2\%) persisted in their treatment for $>12$ months from their first visit to the General Memory Clinic, 9 (6.3\%) persisted for 6-12 months, and $21(14.6 \%)$ persisted for $<6$ months. All the compliant patients persisted in treatment for $>12$ months, whereas $60 \%$ of the noncompliant patients persisted for $<6$ months.

\section{DISCUSSION}

The majority of patients were compliant to the use of $A D$ medications, as measured by the renewal of their previous prescription within 60 days; about $25 \%$ of the patients were non-compliant to medication use. This finding is consistent with 
a recent study conducted in Taiwan, where the non-compliance rate of AChEls use was $24 \% .{ }^{(20)}$ Similarly, AChEI non-compliance rates ranging from $25 \%$ to $50 \%$ have been reported in other observational studies. ${ }^{(4,9,21)}$ A systematic review also found that compliance to $\mathrm{AD}$ medications ranged from $17 \%$ to $42 \%$ across seven studies. ${ }^{(18)}$ This illustrates the need for compliance assessment in the individual society, as each is determined by its unique sociocultural background.

A mixture of patient-related, therapy-related, conditionrelated and socioeconomic factors were found to influence the compliance level of our included patients. In terms of patient-related factors, age $(p=0.774)$, ethnicity $(p=0.200)$, and gender $(p=0.332)$ were not associated with medication compliance. This is unlike the findings of Borah et al's study, which found that older patients were more likely to be compliant to dementia medications (OR 1.401, p < 0.001). ${ }^{(10)}$ In the same study, male gender was also found to be positively associated with compliance to AD medications (OR 1.175, p < 0.05). ${ }^{(10)}$

Patients with AD are generally older and more susceptible to medication non-compliance issues, ${ }^{(22-24)}$ especially as cognitive impairment progresses. The main reason for non-compliance in our study was patients' and caregivers' perceptions that memory loss is a natural ageing process and that medical treatment for $\mathrm{AD}$ is less important than treatment for other chronic diseases, such as hypertension, diabetes mellitus and dyslipidaemia. This dismissal of AD treatment is supported by studies, which showed that perception has a significant influence on patients' compliance to medications. ${ }^{(25)}$ Furthermore, Singaporeans tend to perceive dementia as part of 'normal ageing' and 'a stigmatised mental illness'; ${ }^{(26)}$ this view is supported by a previous study on perception of dementia in Singapore. ${ }^{(27)}$ Hence, a multidisciplinary approach is necessary to educate patients and their caregivers about the importance of medication compliance and to aid patients in understanding their medical condition. This can be in the form of pharmacists' engagement, where pharmacists play an active role in educating and raising awareness of AD among patients seen at the General Memory Clinic. In Japan, this approach has been shown to be effective in reducing medication non-compliance by $50 \%-70 \%$. ${ }^{(28)}$ Patient and caregiver education can also be achieved through web-based intervention, such as the use of videos to improve understanding of the importance of medication compliance. $^{(28)}$

In terms of therapy-related factors, seven of our patients were non-compliant due to side effects from the AD medications. Of these, six patients suffered gastrointestinal and cerebral side effects from donepezil use. Side effects as a contributing factor for noncompliance has been reported in several studies conducted in Asia and Europe. ${ }^{(29,30)}$ Thus, it may be necessary to explore new drug delivery methods so as to improve medication compliance among AD patients. In the present study, a majority ( $n=32,86.5 \%$ ) of the non-compliant patients were prescribed a once-daily donepezil tablet. Apart from the once-daily normal film-coated donepezil tablet, the donepezil orodispersible tablet has been shown to have a lower incidence of gastric side effects and transdermal rivastigmine has been found to improve adherence. ${ }^{(25)}$ Comparing transdermal patch application of rivastigmine to the capsule formulation, the patch has shown increased tolerance, consistent drug delivery and ease of titration with improved efficacy at lower doses. ${ }^{(25)}$ Better treatment efficacy may indirectly play a role in improving patients' compliance to medication. ${ }^{(31)}$ For instance, Molinuevo and Arranz showed an improvement in compliance to AD medications using patches instead of oral tablets or capsules. ${ }^{(32)}$

Regarding condition-related factors, caregivers and patients with comorbidities perceived comorbidities as being more critical than memory loss and dementia. Cardiovascular comorbidities and other chronic conditions such as diabetes mellitus are managed with several medications, resulting in the issue of polypharmacy in this group of patients. While no direct epidemiological studies for AD patients are available locally, the WiSE study conducted in Singapore suggested that diabetes mellitus, ischaemic heart disease and hypertension may be the common comorbidities among AD patients. ${ }^{(33)}$ In view of this perception, interventions should involve equipping patients and caregivers with knowledge about dementia, the importance of medication compliance and the implications of progressive deteriorating $\mathrm{AD}$. The involvement of caregivers is crucial, especially for severe AD patients, who might not have the autonomy to make decisions regarding treatment and medication use. A systematic review has reported successful interventions in caregiver's education and subsequent improvement in the medication compliance level of AD patients. ${ }^{(18)}$

In the present study, only one patient was non-compliant due to the high cost of medications (socioeconomic factor). Despite the small number, it is important to consider socioeconomic factors as a component for assessment of compliance in $A D$ patients. A study conducted in India found that the high cost of medications was associated with non-compliance. ${ }^{(19)}$ In terms of healthcare financing, Singapore adopts a co-payment model, with a medical savings account. ${ }^{(34)}$ In Singapore, the amount of medication subsidies depends on the financial classification of the medications; medications under the Standard Drug List (SDL) or Medication Assistance Fund are heavily subsidised by the government, while medications classified as 'non-standard' are not subsidised. ${ }^{(35)}$ While donepezil is an SDL drug, rivastigmine and memantine remain as non-standard drugs. Apart from medication costs, dementia treatment itself incurs high societal and direct/indirect individual costs, as revealed by a systematic review. ${ }^{(36)}$ In 2013, the total cost of dementia in Singapore was estimated at SGD 532 million, with an annual cost per person of approximately SGD 10,245. ${ }^{(37)}$ This high societal cost will inevitably translate to high treatment and medication costs for patients and their caregivers. This suggests the need for costeffectiveness analysis to be conducted in Singapore, as well as interventions to improve medication compliance that would take into account both direct and indirect costs.

The present study showed that around $60 \%$ of non-compliant patients persisted in treatment for less than six months, with a mean duration of treatment of around ten months. This was in marked contrast with the results of a Taiwanese study, which showed a mean treatment duration of around 28 months. ${ }^{(20)}$ 
This contrast illustrates the fact that it is essential to analyse and understand the factors leading to non-compliance that are unique to each society.

We have identified several factors leading to medication non-compliance, including patient and caregiver perceptions, side effects of medications, treatment efficacy and cost of medications. We opine that a multifaceted approach will be most effective at improving compliance in patients with multiple risk factors influencing their behaviour toward medication use. ${ }^{(21)}$ This can be conducted in the form of a collaborative care model involving the following stakeholders: ${ }^{(38)}$ (a) physicians who optimise medications and other behavioural therapies to ensure optimal treatment efficacy for the patient; (b) clinical psychologists who educate the patients on the importance of AD treatment and its implications; (c) pharmacists who manage the drug regimen, educate patients on side-effect management, and monitor medication use through building rapport with the patient and caregivers; and (d) social workers who manage the financial aspects of treatment and medications for AD patients with financial difficulties.

In 2017, a group of pharmacists who implemented a collaborative care model in the management of diabetes mellitus in Singapore reported an improvement in several humanistic and economic outcomes. ${ }^{(39)}$ This form of collaborative care model can be adopted in the management of AD patients with the aims of concurrently improving medication compliance as well as humanistic/economic outcomes, the latter of which play an indirect role in ensuring that AD patients comply with medication use.

The present study was not without limitations. Firstly, as the study measured medication compliance indirectly based on the renewal of previous prescription within 60 days, we were unable to determine the patients' actual use of AD medications. However, we opine that the duration of 60 days remained reasonable in our study context, as it is the average follow-up duration between visits for dementia patients at National University Hospital, and previous studies have also adopted durations that were specific to their study context. ${ }^{(15-17,40)}$ Secondly, the study was limited by incomplete information from hospital records on patients' lifestyle and social history, and other potential confounders. For example, patients might have obtained their prescription from other healthcare institutions, which we do not have data on. Patients might have also accumulated medications prescribed after hospitalisation and hence did not fill their outpatient prescription. To minimise the effect of this limitation, we contacted caregivers via the telephone to obtain information about the factors leading to the patients' non-compliance to medications. In addition, the patients' physical case notes were reviewed to ensure minimal missing data. Thirdly, the sample size of our study may not be large enough to yield small but statistically significant associations with demographic factors. Furthermore, patients discharged from regular follow-up with the General Memory Clinic (either to another clinical provider or outpatient community care) were considered to be compliant to medication use. This might have resulted in an underestimation of non-compliance, because subjects who became non-compliant while under the care of another clinical provider or outpatient community clinic would not have been counted as non-compliant subjects. In view of this limitation, compliance was tracked to the time-point at which the patient was discharged to another clinical provider.

Despite these limitations, the present study, being the first such study in Singapore, provides a good descriptive overview of factors leading to non-compliance to $A D$ medications. It may serve to inform future practice in Singapore, catering to the needs generated by the unique sociocultural background of Singaporeans. Moving forward, this study may also provide a strong foundation for future behavioural and needs assessment of caregivers of $\mathrm{AD}$ patients, as well as for evaluation of the clinical, humanistic and economic outcomes of non-compliant AD patients.

In conclusion, AD significantly affects the patient's quality of life, ${ }^{(25)}$ and hence compliance to medications for dementia is of utmost importance. In this study, we determined the reasons for non-compliance to AChEls and NMDA receptor antagonist, as well as the time-point of treatment discontinuation. The results showed no association between demographic characteristics and compliance to medication use, whereas the perceptions about dementia among patients and their caregivers played a major role. Further studies should be conducted to identify other reasons for non-compliance to AD medications. A multidisciplinary approach in educating patients and their caregivers about the importance of treatment and medication use is crucial, and the involvement of pharmacists should be considered, as it has been shown to improve compliance to medications. ${ }^{(41)}$

\section{REFERENCES}

1. Tan WJ, Hong S, Luo N, Lo TJ, Yap P. The lay public's understanding and perception of dementia in a developed Asian nation. Dement Geriatr Cogn Disord Extra 2012; 2:433-44.

2. Marseille DM, Silverman DH. Recognition and treatment of Alzheimer's disease: a case-based review. Am J Alzheimers Dis Other Demen 2006; 21:119-25.

3. Catindig JA, Venketasubramanian N, Ikram MK, Chen C. Epidemiology of dementia in Asia: insights on prevalence, trends and novel risk factors. J Neurol Sci 2012; 321:11-6.

4. Herrmann N, Binder C, Dalziel W, Smyth S, Camacho F. Persistence with cholinesterase inhibitor therapy for dementia: an observational administrative health database study. Drugs Aging 2009; 26:403-7.

5. Birks J. Cholinesterase inhibitors for Alzheimer's disease. Cochrane Database Syst Rev 2006; (1):CD005593.

6. Solas M, Puerta E, Ramirez MJ. Treatment options in Alzheimer's disease: the GABA story. Curr Pharm Des 2015; 21:4960-71.

7. Riepe M, Weinman J, Osae-Larbi J, et al. Factors associated with greater adherence to and satisfaction with transdermal rivastigmine in patients with Alzheimer's disease and their caregivers. Dement Geriatr Cogn Disord 2015; 40:107-19.

8. Tian H, Abouzaid S, Chen W, Kahler KH, Kim E. Patient adherence to transdermal rivastigmine after switching from oral donepezil: a retrospective claims database study. Alzheimer Dis Assoc Disord 2013; 27:182-6.

9. Haider B, Schmidt R, Schweiger C, et al. Medication adherence in patients with dementia: an Austrian cohort study. Alzheimer Dis Assoc Disord 2014; 28:128-33

10. Borah B, Sacco P, Zarotsky V. Predictors of adherence among Alzheimer's disease patients receiving oral therapy. Curr Med Res Opin 2010; 26:1957-65.

11. Brady R, Weinman J. Adherence to cholinesterase inhibitors in Alzheimer's disease: a review. Dement Geriatr Cogn Disord 2013; 35:351-63.

12. Lužný J, Ivanová K, Juríčková L. Non-adherence in seniors with dementia: a serious problem of routine clinical practice. Acta Medica (Hradec Kralove) 2014; 57:73-7.

13. Elliot RA, Goeman D, Beanland C, Koch S. Ability of older people with dementia or cognitive impairment to manage medicine regimens: a narrative review. Curr 
Clin Pharmacol 2015; 10:213-21.

14. World Health Organization. Adherence to long-term therapies: evidence for action. Available at: http://apps.who.int/iris/bitstream/10665/42682/1/9241545992.pdf. Accessed June 14, 2016.

15. Amuah JE, Hogan DB, Eliasziw M, et al. Persistence with cholinesterase inhibitor therapy in a population-based cohort of patients with Alzheimer's disease. Pharmacoepidemiol Drug Saf 2010; 19:670-9.

16. Singh G, Thomas SK, Arcona S, Lingala V, Mithal A. Treatment persistency with rivastigmine and donepezil in a large state medicaid program. J Am Geriatr Soc 2005; 53:1269-70.

17. Suh DC, Thomas SK, Valiyeva E, Arcona S, Vo L. Drug persistency of two cholinesterase inhibitors: rivastigmine versus donepezil in elderly patients with Alzheimer's disease. Drugs Aging 2005; 22:695-707.

18. El-Saifi N, Moyle W, Jones C, Tuffaha H. Medication adherence in older patients with dementia: a systematic literature review. J Pharm Prac 2017 Jan 1. [Epub ahead of print]

19. Dhikav V, Singh P, Anand KS. Medication adherence survey of drugs useful in prevention of dementia of Alzheimer's type among Indian patients. Int Psychogeriatr 2013; 25:1409-13.

20. Chang YP, Yang $\mathrm{CH}$, Chou MC, Chen $\mathrm{CH}$, Yang YH. Clinical compliance to donepezil in treating Alzheimer's disease in Taiwan. Am J Alzheimers Dis Other Demen 2015; 30:346-51.

21. Blais L, Kettani FZ, Perreault S, et al. Adherence to cholinesterase inhibitors in patients with Alzheimer's disease. J Am Geriatr Soc 2009; 57:366-8.

22. Kairuz T, Bye L, Birdsall R, et al. Identifying compliance issues with prescription medicines among older people: a pilot study. Drugs Aging 2008; 25:153-62.

23. Tordoff JM, Bagge ML, Gray AR, Campbell AJ, Norris PT. Medicine-taking practices in community-dwelling people aged $>$ or $=75$ years in New Zealand. Age Ageing 2010; 39:574-80.

24. Smith D, Lovell J, Weller C, et al. A systematic review of medication nonadherence in persons with dementia or cognitive impairment. PLoS One 2017; 12:e0170651.

25. Brady R, Weinman J. Adherence to cholinesterase inhibitors in Alzheimer's disease: a review. Dement Geriatr Cogn Disord 2013; 35:351-63.

26. Cipriani G, Borin G. Understanding dementia in the sociocultural context: a review. Int J Soc Psychiatry 2015; 61:198-204.

27. World Health Organization. The epidemiology and impact of dementia. Current state and future trends. Available at: http://www.who.int/mental_health/ neurology/dementia/dementia_thematicbrief_epidemiology.pdf. Accessed June 1, 2016.
28. Gupta V, Hincapie AL, Frausto S, Bhutada NS. Impact of a web-based intervention on the awareness of medication adherence. Res Social Adm Pharm 2015; 12:926-36.

29. Pariente A, Fourrier-Réglat A, Bazin F, et al. Effect of treatment gaps in elderly patients with dementia treated with cholinesterase inhibitors. Neurology 2012; 78:957-63.

30. Olazarán J, Navarro E, Rojo JM. Persistence of cholinesterase inhibitor treatment in dementia: insights from a naturalistic study. Dement Geriatr Cogn Dis Extra 2013; 3:48-59.

31. Campbell NL, Perkins AJ, Gao S, et al. Adherence and tolerability of Alzheimer's disease medications: a pragmatic randomized trial. J Am Geriatr Soc 2017; 65:1497-504.

32. Molinuevo JL, Arranz FJ. Impact of transdermal drug delivery on treatment adherence in patients with Alzheimer's disease. Expert Rev Neurother 2012; 12:31-7.

33. Subramaniam M, Chong SA, Vaingankar JA, et al. Pervalence of dementia in people aged 60 years and above: results from the WiSE study. J Alzheimers Dis 2015; 45:1127-38.

34. McKee M, Busse R. Medical savings accounts: Singapore's non-solution to healthcare costs. BMJ 2013; 347:f4797.

35. Ministry of Health, Singapore. Drug subsidies and schemes. Available at: https://www.moh.gov.sg/content/moh_web/home/costs_and_financing/ schemes_subsidies/drug_subsidies.html. Accessed July 1, 2017.

36. Knapp M, lemmi V, Romeo R. Dementia care costs and outcomes: a systematic review. Int J Geriatr Psychiatry 2013; 28:551-61.

37. Abdin E, Subramaniam M, Achilla E, et al. The societal cost of dementia in Singapore: results from the WiSE study. J Alzheimers Dis 2016; 51:439-49.

38. Forsyth K, Melton J, Raber C, Burke JP, Piersol CV. Scholarship of practice in the care of people with dementia: creating the future through collaborative efforts. Occup Ther Health Care 2015; 29:429-41.

39. Siaw MYL, Ko Y, Malone DC, et al. Impact of pharmacist-involved collaborative care on the clinical, humanistic and cost outcomes of high-risk patients with type 2 diabetes (IMPACT): a randomized controlled trial. J Clin Pharm Ther 2017; 42:475-82.

40. Umegaki H, Itoh A, Suzuki Y, Nabeshima T. Discontinuation of donepezil for the treatment of Alzheimer's disease in geriatric practice. Int Psychogeriatr 2008; 20:800-6.

41. Nanaumi $Y$, Onda M, Tsubota K, et al. Effectiveness of pharmacists' comprehensive assessment of medication profiles in dementia patients. Yakugaku Zasshi 2015; 135:1057-67. 